\title{
The MAPK and PI3K pathways mediate CNTF-induced neuronal survival and process outgrowth in hypothalamic organotypic cultures
}

\author{
Jason M. Askvig • John A. Watt
}

Received: 14 November 2014 / Accepted: 5 February 2015 /Published online: 20 February 2015

(C) The Author(s) 2015. This article is published with open access at Springerlink.com

\begin{abstract}
While collateral sprouting has been shown to occur in a variety of neuronal populations, the factor or factors responsible for mediating the sprouting response remain largely un-defined. There is evidence indicating that ciliary neurotrophic factor (CNTF) may play an important role in promoting neuronal survival and process outgrowth in neuronal phenotypes tested to date. We previously demonstrated that the astrocytic Jak-STAT pathway is necessary to mediate CNTF-induced oxytocinergic (OT) neuronal survival; however, the mechanism (s) of CNTF-mediated process outgrowth remain unknown. Our working hypothesis is that CNTF mediates differential neuroprotective responses via different intracellular signal transduction pathways. In order to test this hypothesis, we utilized stationary hypothalamic organotypic cultures to assess the contribution of the MAPK-ERK and PI3-AKT pathways to OT neuron survival and process outgrowth. Our results demonstrate that the MAPK-ERK $1 \frac{1}{2}$ pathway mediates CNTFinduced neuronal survival. Moreover, we show that inhibition of the p38-, JNK-MAPK, and mTOR pathways prevents loss OT neurons following axotomy. We also provide quantitative evidence indicating that CNTF promotes process outgrowth of OT neurons via the PI3K-AKT pathway. Together, these data indicate that distinct intracellular
\end{abstract}

\section{J. M. Askvig}

Department of Biology, Concordia College, Moorhead,

MN 56562, USA

e-mail: jaskvig@cord.edu

\section{J. A. Watt $(\bowtie)$}

Department of Basic Sciences, University of North Dakota School of

Medicine and Health Sciences, Room 1701 Stop 9037, 501 N

Columbia Road, Grand Forks, ND 58203, USA

e-mail: john.watt@med.und.edu signaling pathways mediate diverse neuroprotective processes in response to CNTF.

Keywords PI3K $\cdot$ AKT $\cdot$ MAPK $\cdot \mathrm{STAT} 3 \cdot \mathrm{NFkB} \cdot \mathrm{CNTF}$. Axonal regeneration $\cdot$ Neuronal survival $\cdot$ Organotypic culture

$\begin{array}{ll}\text { Abbreviations } \\ \text { CNTF } & \text { Ciliary neurotrophic factor } \\ \text { CNTFR } \alpha & \text { Ciliary neurotrophic factor receptor alpha } \\ \text { gp130 } & \text { Glycoprotein } 130 \\ \text { LIFRß } & \text { Leukocyte inhibitory factor receptor beta } \\ \text { OT } & \text { Oxytocinergic } \\ \text { PVN } & \text { Paraventricular nucleus } \\ \text { SON } & \text { Supraoptic nucleus } \\ \text { VP } & \text { Vasopressinergic }\end{array}$

\section{Introduction}

Collateral axonal sprouting has been shown to occur in a variety of neuronal populations within the central nervous system although the factors responsible for mediating axonal sprouting are still largely ill-defined. However, there is growing evidence indicating that ciliary neurotrophic factor (CNTF) plays an important role. For example, CNTF has been shown to promote axonal sprouting of hypothalamic magnocellular neurons in vitro (Askvig et al. 2013; Vutskits et al. 1998) and motor neuron sprouting in vivo (Gurney et al. 1992; Guthrie et al. 1997; Kwon and Gurney 1994; Oyesiku and Wigston 1996; Siegel et al. 2000; Simon et al. 2010; Ulenkate et al. 1994; Wright et al. 2007; Xu et al. 2009). Moreover, CNTF promotes the survival of magnocellular neurons in the paraventricular nucleus $(\mathrm{PVN})$ and supraoptic nucleus (SON) of the magnocellular neurosecretory system in organotypic cultures (Askvig et al. 2013; House et al. 2009; 
Rusnak et al. 2002; Rusnak et al. 2003; Vutskits et al. 1998; Vutskits et al. 2003).

To induce a cellular response, CNTF first binds to a threepart receptor complex consisting of the ligand-specific binding subunit CNTF receptor alpha (CNTFR $\alpha$ ), which is attached to the membrane via a glycosylphosphatidylinositol (GPI) linkage. The CNTF-CNTFR $\alpha$ complex then interacts with glycoprotein 130 (gp130) and leukemia inhibitory factor receptor beta (LIFRß) (Davis et al. 1993; Ip et al. 1993; Ip et al. 1992; Schuster et al. 2003) to form a functional transmembrane signaling complex. We have demonstrated previously that astrocytes within the SON are immunoreactive for CNTFR $\alpha$, LIFR $\beta$, gp130 and CNTF protein (Askvig et al. 2012; Watt et al. 2006). However, magnocellular neurons are immunoreactive for CNTFR $\alpha$ and gp130 (Askvig et al. 2012; Watt et al. 2009) but not LIFRß indicating that the astrocytes but not magnocellular neurons of the SON are activated directly by CNTF (Davis et al. 1993). In support of this observation, we have shown that pressure injection of exogenous rat recombinant (rCNTF) into the SON in vivo results in phosphorylation of STAT3 in astrocytes but not magnocellular neurons (Askvig et al. 2013). CNTF-induced activation of the Jak-STAT pathway mediates a significant increase in survival of axotomized magnocellular neurons in organotypic culture which was reduced by pharmacological inhibition of the JakSTAT pathway (Askvig et al. 2013). In addition, we observed an extensive outgrowth of neuronal processes originating from oxytocinergic (OT) neurons in the SON and PVN in response to rrCNTF (Askvig et al. 2013). Together, these data indicate that CNTF-induced survival of OT magnocellular neurons is mediated through a paracrine interaction with astrocytes. Although the specific intracellular pathway (s) which mediate CNTF-induced process outgrowth remain unknown, others have demonstrated differential effects of the Jak-STAT, PI3-AKT and MAPK-ERK pathways in mediating neuronal survival versus process outgrowth (Alonzi et al. 2001; Dolcet et al. 2001; Ozog et al. 2008; Sango et al. 2008).

Our working hypothesis is that CNTF mediates neuronal survival and process outgrowth via different intracellular signal transduction pathways, respectively. In order to test this hypothesis, we utilized stationary hypothalamic organotypic cultures, as initially developed by Stoppini et al. (1991), to assess the contribution of the MAPK, NFkB and PI3-AKT pathways to magnocellular neuron survival and process outgrowth in the SON and PVN. Organotypic cultures exhibit several advantages over other in vitro culture systems primarily because of the preservation of the in vivo cytoarchitecture and the use of fully differentiated neurons (House et al. 1998; Vutskits et al. 1998). Furthermore, the ability to directly manipulate the culture media with growth factors and pharmacological agents and assess magnocellular neuron survival in hypothalamic organotypic cultures facilitates analysis of pathway-mediated cellular events more rapidly than can be achieved using in vivo injury model systems. However, as with all in vitro systems when interpreting experimental results consideration must be given to alteration of cellular activities specific to the tissue preparation and culture conditions used which may affect experimental outcomes. Nevertheless, stationary organotypic cultures provide a unique and powerful tool for exploring the factors and mechanisms through which specific central nervous systems respond to injury

\section{Materials and methods}

Animals

Pregnant Sprague Dawley rats (E15) were purchased from Harlan Laboratories (Minneapolis, MN). All rats were housed in the University of North Dakota Center for Biomedical Research Facility, an AAALAC accredited facility, under a $12 \mathrm{~L}: 12 \mathrm{D}$ light cycle with ad lib access to lab chow and tap water throughout the investigations. Experimental protocols utilized in these studies followed the guidelines in the NIH guide for the care and use of laboratory animals and were approved by the UND Institutional Animal Care and Use Committee. All efforts were made to minimize the numbers of animals used in this study and their suffering.

Stationary hypothalamic organotypic cultures

Organotypic cultures were prepared as previously described (Askvig et al. 2013). Briefly, 6-day-old Sprague Dawley rat pups were decapitated and their brains were removed and placed in chilled Geys Balanced Salt Solution (Gibco, Grand Island, NY) enriched with glucose (5 mg/ml; Sigma). The brains were then trimmed to remove exterior cortical material and $350 \mu \mathrm{m}$ coronal sections obtained using a Mcllwain Tissue Chopper (Stoelting). The sections containing the magnocellular neurosecretory system nuclei were placed in chilled Geys Balanced Salt Solution and then trimmed dorsal to the third ventricle and lateral to the SON under a dissecting microscope. Sections from each animal were then placed on a single Millicell-CM filter insert (pore size $0.4 \mu \mathrm{m}, 30 \mathrm{~mm}$ diameter; Millipore, Bedford, MA) and each filter insert was then placed in a $35 \times 10 \mathrm{~mm}$ Petri dish containing 1.1-1.2 ml of culture media for the experimental period.

Media and incubations

The culture media was made fresh at the beginning of every experiment and consisted of Eagle's Basal Medium with Earle's salts ( $50 \%$; Gibco), heat inactivated horse serum (25\%; Gibco), Hank's balanced salt solution (25\%; Gibco), glucose $(0.5 \%$; Sigma), penicillin/streptomycin ( 25 units $/ \mathrm{ml}$; Gibco), and glutamine (1.0 mM; Gibco). The osmolality and 
$\mathrm{pH}$ of the culture media were measured from the stock media solution every $48 \mathrm{~h}$ using a Wescor vapor pressure osmometer (Wescor 5500; Logan, UT) and a mini pH meter (IQ Scientific Instruments, Loveland, CO), respectively. Our analysis demonstrated that the media osmolality was maintained at $310.5 \pm$ $0.45 \mathrm{mOsm} / 1$ and the $\mathrm{pH}$ was at $8.2 \pm 0.03$ throughout the experimental period. Incubation of the cultures was stationary in $5 \% \mathrm{CO}_{2}$-enriched air at $35{ }^{\circ} \mathrm{C}$ for the entire experimental period.

Hypothalamic slices were cultured in the presence or absence of rrCNTF (\#C3835, lot \#080 M1730 or \#091 M1403, Sigma) for 14 days. All groups had their culture media replaced every $48 \mathrm{~h}$ and always received fresh additions of rrCNTF. Inhibition experiments were performed by administering the inhibitor in the absence of rrCNTF for $1 \mathrm{~h}$ prior to treatment of the cultures followed by replacement with media containing the inhibitor plus rrCNTF for the duration of the experimental period. Additional control cultures received only the inhibitor for the entire experimental period. Multiple inhibitors, which had distinct mechanisms of action, were used to inhibit the pathways. The concentrations used for each inhibitor was determined from previous reports of others indicating optimal working concentrations of inhibitors used in primary cell cultures (Phulwani et al. 2008; Su et al. 2011; Wang et al. 2008), or when available, in organotypic cultures (Greenwood and Bushell 2010; Luo et al. 2007; Marwarha et al. 2010; Ohnishi et al. 2010; Rusnak and Gainer 2005; Zamin et al. 2006). Briefly, inhibition of various components of the MAPK pathway were inhibited with U0126, PD98059, PD184352, SP600125, and SB203580, the PI3K-AKT pathway was inhibited with LY294002 and wortmannin, mTOR signaling was inhibited utilizing rapamycin and torin- 1 , and the NFKB transcription factor was inhibited by bay 11-7082 and sc-514 (Table 1).
Organotypic culture immunohistochemistry

Following experimental periods, the explants were prepared for immunohistochemistry with fixation in $4 \%$ paraformaldehyde (Sigma) in $0.1 \mathrm{M}$ phosphate buffer for $1.5 \mathrm{~h}$. For immunohistochemical analysis, sections were washed with PBS-T in $3 \times 10 \mathrm{~min}$ intervals before and after all incubations. For single-label peroxidase immunohistochemistry, endogenous peroxidase activity and non-specific staining were prevented by treatment with $0.3 \% \mathrm{H}_{2} \mathrm{O}_{2}$ (Sigma) followed by incubation in blocking buffer $(10 \%$ normal horse serum containing $0.3 \%$ Triton X-100) for $1 \mathrm{~h}$. The explants were then incubated for $36 \mathrm{~h}$ at $4{ }^{\circ} \mathrm{C}$ in a highly specific monoclonal mouse antibody against oxytocin (OT)-neurophysin (PS 38, 1:500; a gift from Dr. Harold Gainer). This antibody was first characterized by Ben-Barak et al. (1985) and has been shown to label specifically OT neurons and their processes within stationary organotypic cultures prepared from rat hypothalamus (House et al. 2009; House et al. 2006; House et al. 1998; Rusnak et al. 2002; Rusnak et al. 2003; Shahar et al. 2004; Vutskits et al. 1998; Vutskits et al. 2003). Next the cultures were incubated in horse anti-mouse biotinylated secondary antibody (1:500; Vector), followed by avidin-biotin complex (ABC; $10 \mu \mathrm{l} / \mathrm{ml}$ in PBS; Vector ABC Elite kit) for $1 \mathrm{~h}$ at room temperature. Bound antibodies were visualized using $0.05 \%$ diaminobenzidine (DAB, Sigma) in PBS developed through the glucoseoxidase method (Itoh et al. 1979). The hypothalamic slices were then removed from their filters and placed directly on gelatin coated slides. All slides were then dehydrated in increasing concentrations of alcohol followed by xylene and coverslips mounted with Permount (Fisher, Pittsburgh, PA). All images were captured using an Olympus BX-51 light microscope with attached DP-71 color camera and dedicated software. Montage images were prepared for reproduction using the 'photomerge' option in Adobe Photoshop CS3.
Table 1 Pharmacological inhibitors used in this study

\begin{tabular}{lll}
\hline Inhibitor (Concentration used) & Site of action & Source/Cat \# \\
\hline Bay 11-7082 $(15,30 \mu \mathrm{M})$ & IKB $\alpha$ & Calbiochem \# 196870 \\
LY294002 $(15 \mu \mathrm{M})$ & PI3K & Calbiochem \#440202 \\
LY303511 $(15 \mu \mathrm{M})$ & Negative control for LY294002. & Calbiochem \# 440203 \\
PD184352 $(5 \mu \mathrm{M})$ & MEK $1 / 2$ & Santa Cruz \#sc-202759 \\
PD98059 $(5 \mu \mathrm{M})$ & MEK $1 / 2$ and MEK5 & Calbiochem \#513000 \\
Rapamycin $(10 \mu \mathrm{M})$ & mTORC1 & Calbiochem \#553210 \\
SB203580 $(75 \mu \mathrm{M})$ & p38 MAPK & Calbiochem \# 559389 \\
SC-514 $(20 \mu \mathrm{M})$ & IKK-2(ß) & Calbiochem \# 401479 \\
SP600125 JNKII Inhibitor $(50 \mu \mathrm{M})$ & c-Jun N-terminal kinase (JNK) & Calbiochem \# 420119 \\
Torin-1 $(500 \mathrm{nM})$ & mTORC1 and mTORC2 & Tocris (R\&D Systems) \#4247 \\
U 0124 $(1 \mu \mathrm{M})$ & Negative control for U 0126. & Calbiochem \# 662006 \\
U 0126 $(1 \mu \mathrm{M})$ & MEK $1 / 2$ and MEK5 & Calbiochem \# 662005 \\
Wortmannin $(\mathrm{KY} 12420)(1 \mu \mathrm{M})$ & PI3K & Calbiochem \# 681675 \\
\hline
\end{tabular}


Magnocellular neuronal counts

The slides containing the immunoreactive explant culture slices were coded by a third party blind to the experimental conditions. In order to obtain the total number of neurons in the PVN and SON, immunoreactive cells were counted using a drawing tube attached to an Olympus BX51 microscope. The values used in statistical analysis represent the total number of immunoreactive neurons for each nuclei of one neonatal hypothalami (i.e., one filter insert) and it was the mean of two individual's independent neuronal counts that were used as the group mean for statistical analysis as described below.

Quantitative analysis of process density

In order to quantify the extent of OT-immunoreactive process outgrowth originating from the SON in organotypic cultures, we utilized a previously described stereological analysis technique first developed for quantification of neurite outgrowth from chick dorsal root ganglion (Bilsland et al. 1999) and modified for our culture system. Slides coded by a third party blind to the experimental conditions were viewed at $10 \times$ magnification using an Olympus BX51 microscope. In order to ensure consistency across groups, the SON was placed in the lower center frame of the picture $(1360 \times 1024$ image size $)$ and the frame digitally captured. MCID image analysis software (Cambridge, England) was used to quantify the area occupied by OT-immunoreactive processes using an automatic target detection and measurement feature that quantifies the area in pixels occupied by a target according to the defined target criteria (Bilsland et al. 1999). The target criteria are set combining the optical density and spatial characteristics of the target. Once the density was set, any pixel falling within this range in the image was automatically counted. A full image scan of the micrograph was performed which gave the proportional area of the entire micrograph that was occupied by OTimmunoreactivity (total proportional area). Next, the density was determined for the immunoreactive somata, which gave the somata proportional area. The somata proportional area was subtracted from the total proportional area to determine the proportional area that was occupied by OTimmunoreactive processes (process proportional area), which was the value that was utilized in the statistical analysis. As each organotypic culture differs in terms of intensity of immunoreactivity and background, the density was set for each image before quantification was undertaken. Since each SON varies in the amount of magnocellular neurons, we corrected for the number of neurons in the SON by standardizing the process proportional area to the total number of neurons in the SON. Thus, the values utilized for statistical analyses, as described below, represent the ratio of process proportional area to total number of neurons in the SON.
Statistical analysis

Distribution normality of each group of data was tested using the Kolmogorov-Smirnov test (GraphPad InStat, version 3.06 for Windows; San Diego California) and all groups were normally distributed. Statistical differences between groups were compared using one-way ANOVA with Tukey's post hoc test (GraphPad InStat) with $p<0.05$ considered statistically significant. Statistical values are reported in the appropriate figure legends. Results are expressed as the group means \pm SD.

\section{Results}

The MAPK-ERK pathway mediates CNTF-induced OTneuronal survival in organotypic cultures

Three pharmacological inhibitors of the MAPK-ERK pathway were utilized; U0126, PD98059, and PD184352 (Table 1). All inhibit MAPKK (MEK), preventing activation of the immediate downstream target, ERK. U0126 and PD98059 are structurally related, and have been demonstrated to inhibit both MEK $1 / 2$ and MEK5 (Ballif and Blenis 2001; Su et al. 2011), while PD184352 specifically inhibits MEK $1 / 2$ (Bain et al. 2007). Organotypic cultures were also treated with U0124, which is a control molecule for the pharmacological inhibitor, U0126, and does not inhibit MEK activity even at concentrations of $100 \mu \mathrm{M}$ (manufacturer's technical sheet).

Our results indicate that treatment of organotypic cultures with $\mathrm{rrCNTF},(25 \mathrm{ng} / \mathrm{ml}, 14$ days) (Askvig et al. 2013), resulted in a $443 \%$ increase in the number of surviving OT neurons in the SON when compared with control values obtained from cultures maintained in rrCNTF-free media (Fig. 1a). Additionally, a significant $52 \%$ increase in OT neuronal survival was also observed in the PVN (Fig. 1b).

When cultures were treated with $25 \mathrm{ng} / \mathrm{ml} \mathrm{rrCNTF}$ in the presence of U0124, the control molecule, there was no significant difference in the number of surviving OT neurons in the SON or PVN (Fig. 1). In contrast, when the MEK $1 / 2$ and MEK5 pathways were inhibited with $1 \mu \mathrm{M}$ U0126 in the presence of rrCNTF we observed a 55 and $40 \%$ decrease in the number of surviving OT neurons in the SON and PVN, respectively (Fig. 1a and b). Likewise, CNTF treatment in the presence of PD98059 resulted in an 80 and $65 \%$ decrease in the number of surviving OT neurons in the SON and PVN, respectively (Fig. 1b).

When cultures were treated with PD184352 in the presence of rrCNTF we observed an 84 and $53 \%$ decrease in OT neurons in the SON and PVN, respectively (Fig. 1b). When organotypic cultures were treated with inhibitors alone there was no significant difference in the number of OT neurons in the SON or PVN (Fig. 1b) demonstrating that the inhibitors alone did not adversely affect OT neuron survival. Together, 
these data demonstrate that pharmacological inhibition of the MAPK pathway prevented CNTF-induced survival of OT neurons in the SON and PVN.

JNK-MAPK and p38 do not mediate CNTF-induced neuronal survival

For the pharmacological inhibition of the p38-MAPK pathway, we utilized SB203580, which inhibits the p38 $\alpha$ and $\beta$ isoforms, but not the $\gamma$ and $\delta$ isoforms, and does not inhibit any of the JNK- or ERK-MAPK isoforms (English and Cobb 2002; Greenwood and Bushell 2010; Lee et al. 1994). Our analysis demonstrated that SB203580 in the presence of rrCNTF did not adversely affect neuronal survival in the SON or PVN (Fig. 2a and b). Conversely, treatment of cultures with inhibitor alone resulted in a 184 and $72 \%$ increase in the number of OT neurons in the SON and PVN, respectively (Fig. 2a and b) suggesting that the p38-MAPK pathway mediates injury-induced cell death of OT neurons.

We next applied SP600125 (JNK Inhibitor II) which has equal potency towards all three of the JNK isoforms (JNK1-3) and cJun (Bennett et al. 2001; Ohnishi et al. 2010). Results indicate that SP600125 in the presence of rCNTF did not adversely affect CNTF-induced neuron survival in the SON (Fig. 2a). However, in the PVN, we observed a $66 \%$ increase

A SON

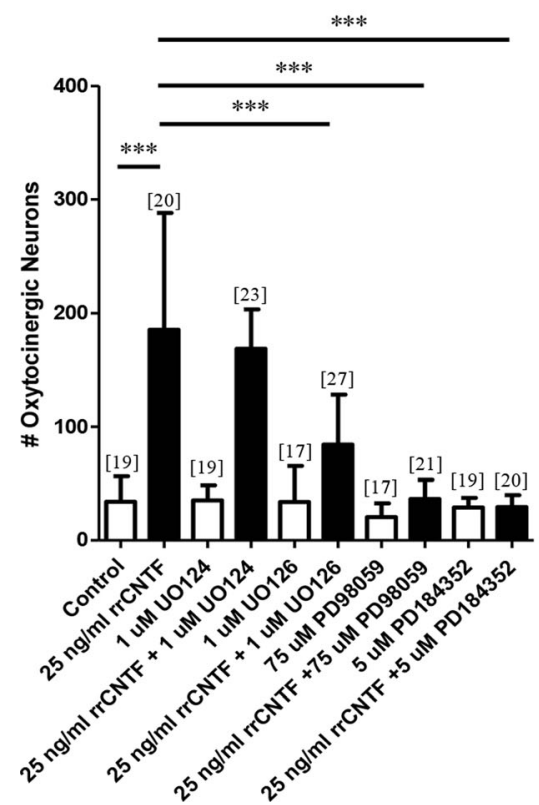

Fig. 1 The MAPK-ERK $1 \frac{1}{2}$ pathway is necessary to mediate the CNTFinduced survival of OT neurons. Immunohistochemical neuronal cell counts demonstrated that exogenous rCNTF promoted the survival of OT neurons (SON: $p<0.0001$; PVN: $p<0.0001$ ) compared to control, while inhibition of the MAPK-ERK $1 \frac{1}{2}$ pathway with U0126 (SON: $p<$ 0.0001 ; PVN: $p<0.0001$ ), PD98059 (SON: $p<0.0001$; PVN: $p<$ in surviving OT neurons following treatment with SP600125 in the presence of rrCNTF. Treatment with SP600125 alone resulted in a $194 \%$ increase in OT neuron survival the SON (Fig. 2a) and by $125 \%$ in the PVN (Fig. 2b).

The PI3K-AKT pathway mediates CNTF-induced process outgrowth

Two pharmacological inhibitors of PI3K were utilized; LY294002, which blocks the ATP binding site of PI3K (Zamin et al. 2006), and wortmannin (KY12420), which blocks the catalytic activity of PI3K (Luo et al. 2007). In addition, LY303511, which contains a single atom substitution in the morpholine ring and does not inhibit PI3K at concentrations up to $100 \mu \mathrm{M}$ (manufacturer's technical sheet) was used as a control for LY294002. We found that treatment with LY303511, LY294002 or wortmannin in the presence of rrCNTF did not result in a significant difference in the number of surviving OT neurons in the SON or PVN, although the number of surviving OT neurons were still significantly elevated from non-CNTF treated control (Fig. 3a and b). When organotypic cultures were treated with inhibitors alone there was no difference in the number of OT neurons in the SON or PVN. Together, these data demonstrate that pharmacological inhibition of the PI3K-AKT pathway does not affect CNTF-

\section{B PVN}

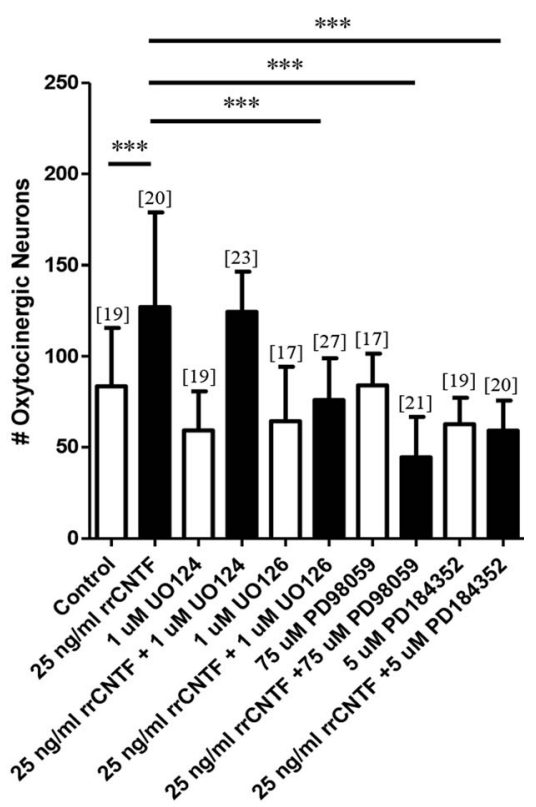

0.0001 ), and PD184352 (SON: $p<0.0001$; PVN: $p<0.0001$ ) significantly reduced the number of surviving OT neurons in the SON (a) and PVN (b) compared to the $25 \mathrm{ng} / \mathrm{ml}$ rrCNTF group. Column bars and error bars represent the mean and SD of [n] groups. PVN, paraventricular nucleus; SON, supraoptic nucleus. ${ }^{* * *} p<0.0001$ 


\section{A SON}

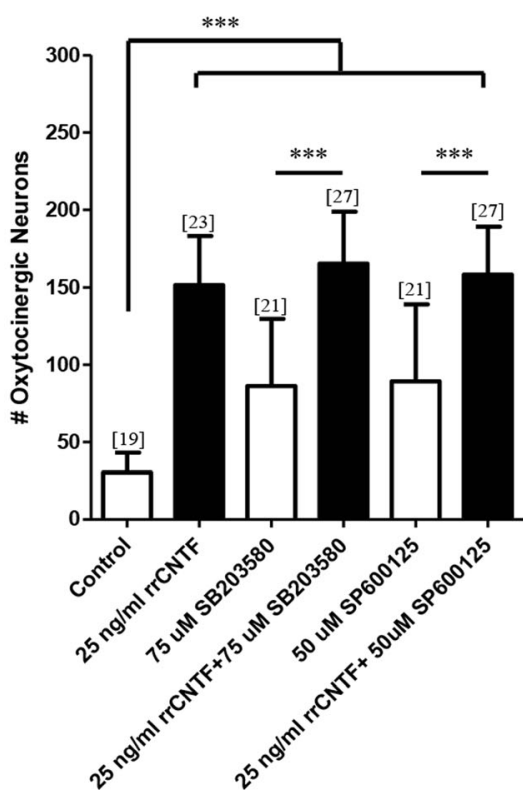

Fig. 2 The p38-and JNK-MAPK pathways are not necessary to mediate the CNTF-induced survival of OT neurons. Immunohistochemical neuronal cell counts demonstrated that exogenous rrCNTF promoted the survival of OT neurons (SON: $p<0.0001$; PVN: $p<0.05$ ) compared to control, while inhibition of the p38- and JNK-MAPK pathways with SB203580 (SON: $p<0.0001$; PVN: $p<0.0001$ ) and SP600125 (SON: $p$ $<0.0001$; PVN: $p<0.0001$ ), respectively, did not affect the number of surviving OT neurons in the SON (a) or PVN (b) compared to the

induced OT neuronal survival in hypothalamic organotypic explant cultures.

When visibly comparing the number of OT neurons between cultures treated with rCNTF (Fig. 3c and inset), and rrCNTF plus LY294002 (Fig. 3d and inset), it is apparent that although there are similar numbers of OT neurons present there are fewer neuronal processes present in the groups receiving PI3K inhibition. Therefore, we utilized quantitative optical densitometric stereological analysis to determine the proportional area of OTimmunoreactive processes in the SON. Figure 4 illustrates the procedure. The value utilized in the statistical analysis was the ratio of the process proportional area to the total number of neurons in the SON. Our results demonstrate that following administration of exogenous rCNTF there was a $112 \%$ increase in the proportional area of OTimmunoreactive processes compared to control (Fig. 4d). LY303511 plus rrCNTF group did not differ from the rrCNTF group (Fig. 4d). However, when the PI3K inhibitors, LY294002 and wortmannin, were administered to the organotypic cultures in the presence of $\operatorname{rrCNTF}$, there was a significant reduction in the proportional area of OTimmunoreactive processes in the SON (Fig. 4d). Moreover, the LY294002 plus rrCNTF group was significantly

\section{B PVN}

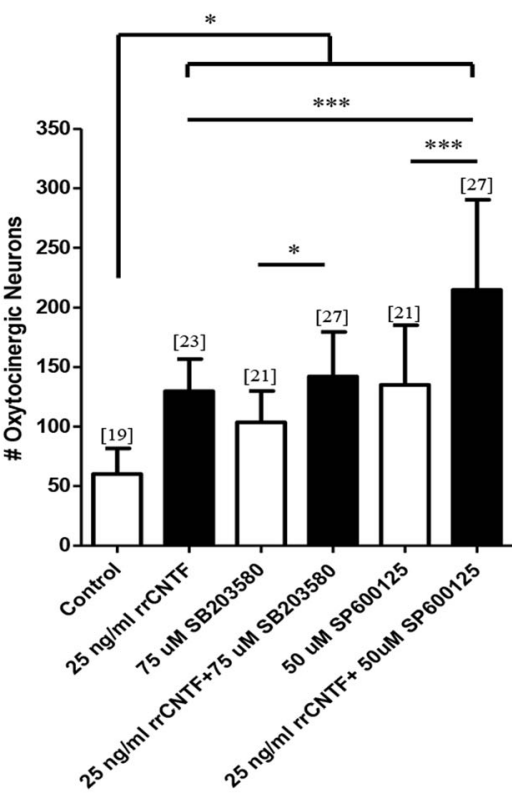

$25 \mathrm{ng} / \mathrm{ml} \mathrm{rrCNTF}$ group. However, when cultures were treated with the inhibitors alone, there was a statistically significant increase in the number of surviving OT neurons in the SON (A; SB203580: $p<0.0001$; SP600125: $p<0.0001)$ and PVN (B; SB203580: $p<0.05$; SP600125: $p$ $<0.0001)$ compared to control. Column bars and error bars represent the mean and $\mathrm{SD}$ of [n] groups. $P V N$ paraventricular nucleus, $S O N$ supraoptic nucleus. ${ }^{*} p<0.05, * * * p<0.0001$

reduced from control values (Fig. 4d). These observations demonstrate that while PI3K signaling does not influence CNTF-mediated neuronal survival, it does mediate CNTFinduced process outgrowth of OT magnocellular neurons in the SON.

mTORC1 and $\mathrm{C} 2$ do not mediate CNTF-induced neuronal survival or process outgrowth

Rapamycin is a selective and potent inhibitor of mTOR complex 1 (mTORC1) (Marwarha et al. 2010), we also utilized torin-1, which is a selective and potent inhibitor for both mTORC1 and mTORC2 (Thoreen et al. 2012; Thoreen et al. 2009; Thoreen and Sabatini 2009). Moreover, torin-1 has been demonstrated to provide more complete inhibition of mTORC1 when compared to rapamycin (Thoreen and Sabatini 2009). Our analysis demonstrated that $10 \mu \mathrm{M}$ rapamycin or $500 \mathrm{nM}$ torin- 1 in the presence of rrCNTF did not result in a statistically significant difference in the number of surviving OT neurons in the SON or PVN (Fig. 5a and b).

When organotypic cultures were treated with rapamycin or torin-1, in the absence of rrCNTF, there was a statistically significant increase in the number of OT neurons in the 
SON and PVN. These results suggest that mTOR signaling may be involved in the injury-induced cell death process in hypothalamic organotypic cultures. However, in contrast to inhibition of PI3K, quantitative optical densitometric analysis demonstrated that pharmacological inhibition of mTOR with rapamycin or torin-1 did not result in a statistically significant difference in the proportional area of OT-immunoreactive processes in the SON (data not shown).
NF-kB does not mediate CNTF-induced neuronal survival or process outgrowth

We utilized two pharmacological agents to inhibit NF-kB. BAY 11-7082, acts by inhibiting I $\mathrm{KB} \alpha$ phosphorylation (Phulwani et al. 2008) while SC-514 targets the IKK complex which consists of three subcomponents, IKK $\alpha, \operatorname{IKK} \beta$, and IKK $\gamma$. SC-514 highly specific for the IKK $\beta$ component and
Fig. 3 The PI3K-AKT pathway is not necessary to mediate the CNTF-induced survival of OT neurons. Immunohistochemical neuronal cell counts demonstrated that exogenous rrCNTF promoted the survival of OT neurons (SON: $p<0.0001$; PVN: $p<0.01$ ) compared to control, while inhibition of the PI3K-AKT pathway with LY294002 (SON: $p=0.6014$; PVN: $p=0.4931) \mathrm{did}$ not affect the number of surviving OT neurons in the SON (a) or PVN (b) compared to $25 \mathrm{ng} / \mathrm{ml}$ rrCNTF. In addition, the presence of wortmannin did not affect the number of surviving OT neurons in the $\mathrm{SON}(\mathrm{A} ; p=0.8791)$; although there was a statistically significant increase in the number of surviving OT neurons in the PVN (B; $p=0.0373)$ compared to the $25 \mathrm{ng} / \mathrm{ml} \mathrm{rrCNTF}$ group When visibly comparing the number of OT neurons between $\operatorname{rrCNTF}(\mathbf{c})$ and the rrCNTF plus LY294002 (d) groups, it is apparent that there are fewer neuronal processes present in the groups receiving PI3K inhibition compared to the rrCNTF group, even though they have the same number of surviving OT neurons (compare insets in C and D). Note that the representative images were obtained from

approximately the same level of the magnocellular neurosecretory system, which is apparent when comparing the III ventricle between the images. Column bars and error bars represent the mean and $\mathrm{SD}$ of [n] groups. $A C C$ accessory nuclei, $P V N$ paraventricular nucleus, $S O N$ supraoptic nucleus. Scale bar C, $\mathrm{D}=300 \mu \mathrm{m}$, inset $=100 \mu \mathrm{m} .{ }^{*} p<$ $0.05, * * p<0.01, * * * p<0.0001$
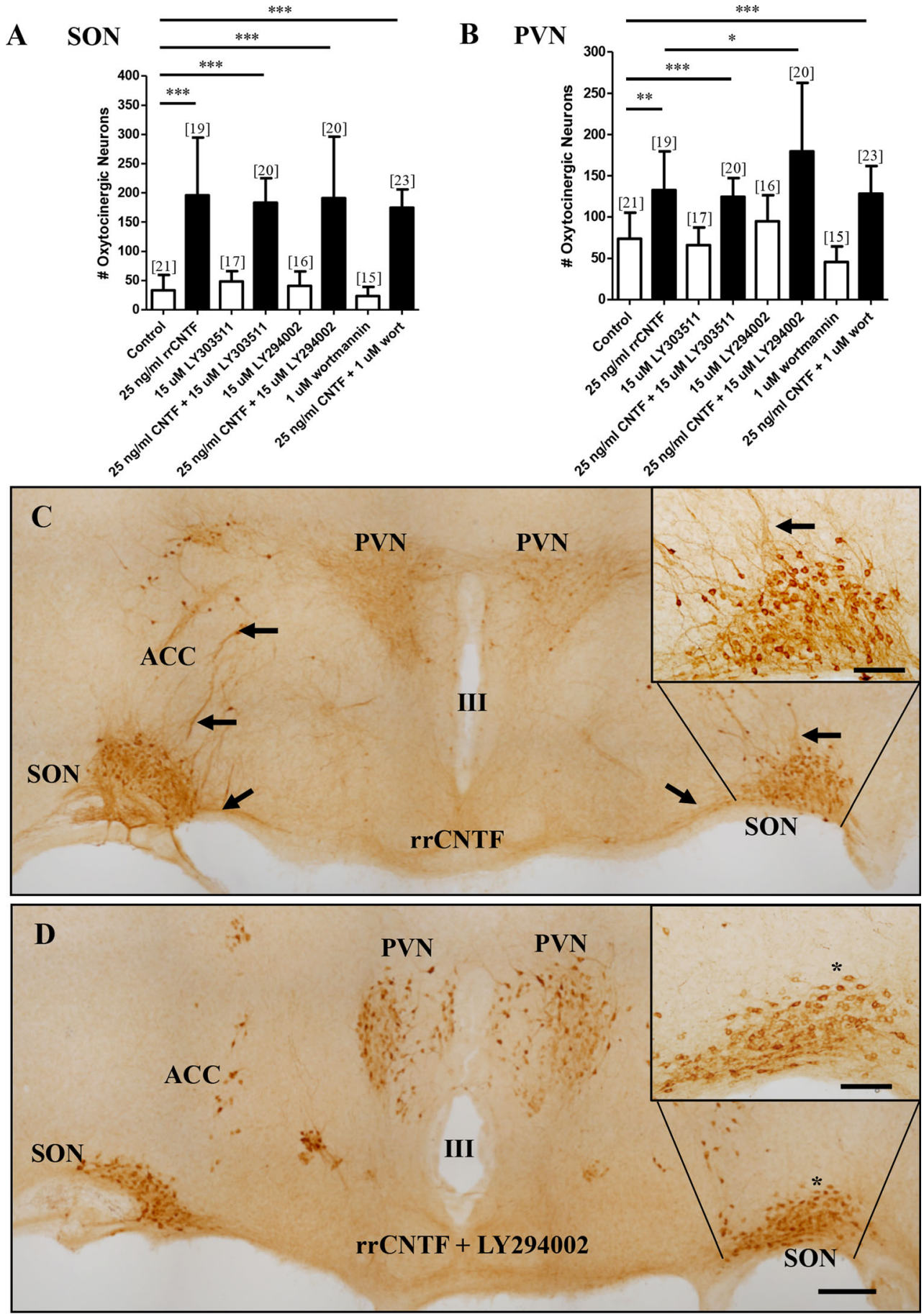

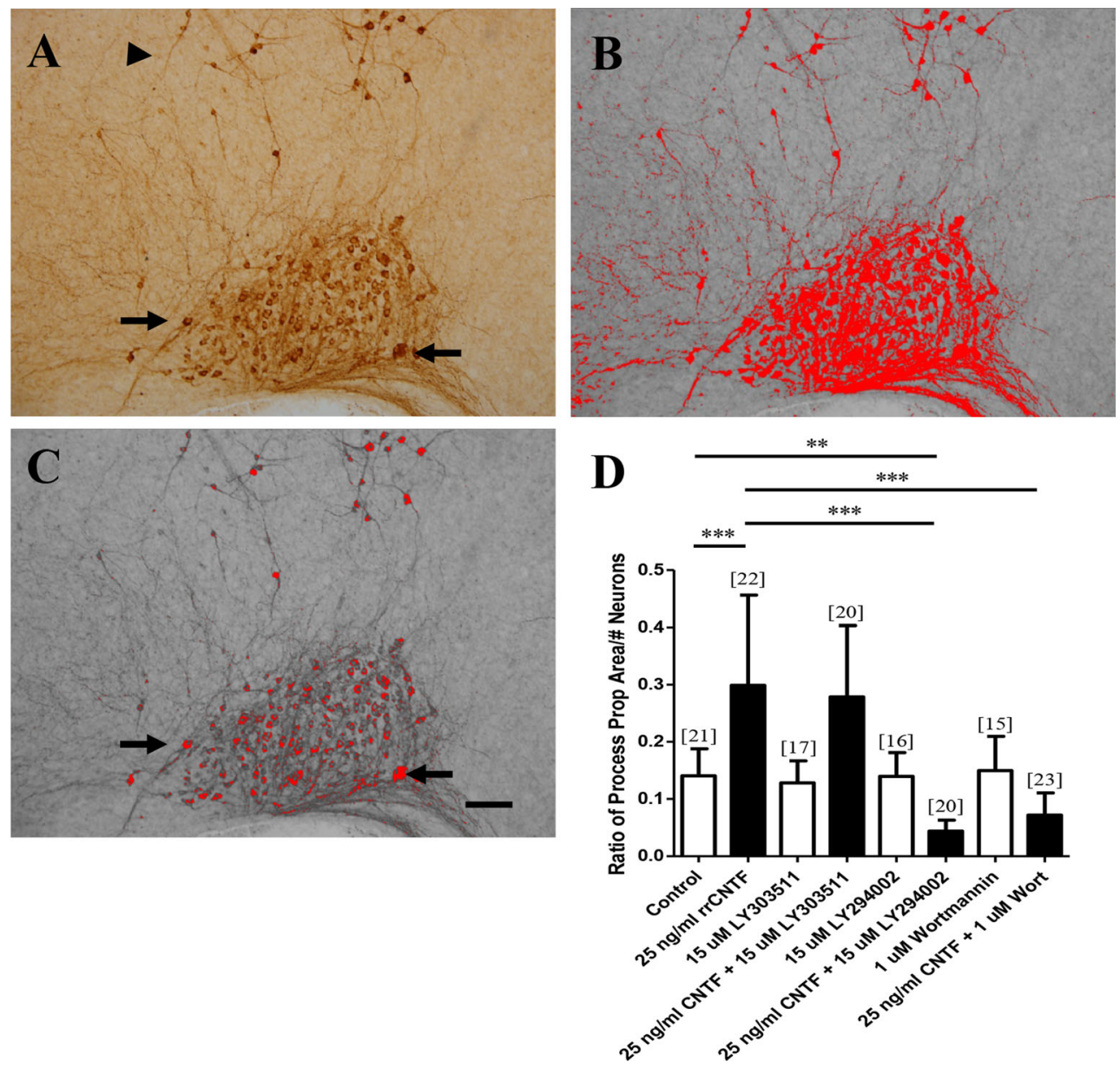

Fig. 4 Inhibition of PI3K reduces the proportional area of OTimmunoreactive processes in the SON. a The image of the SON, captured such that the entire SON is centered in the bottom of the image. b A highlighted SON is shown following setting of the density. The density has been set to highlight the immunoreactive processes and somata, however, note that the analysis is a conservative estimate of the proportional area of neuronal processes because not all of the immunoreactive processes are highlighted (arrowheads, $A, B$ ). c A highlighted SON is shown following the setting of the density to highlight just the immunoreactive somata. Note that similar to their immunoreactive profiles, the nuclei are not highlighted by the density setting (arrows, $A, C$ ). d The ratio of proportional area of OT-immunoreactive processes to total number of neurons in the SON demonstrated that following administration of

shows little effect on the other isoforms in vitro. Our analysis demonstrated that neither bay 11-7082 nor sc-514 in the presence of $25 \mathrm{ng} / \mathrm{ml} \mathrm{rCNTF}$ resulted in a significant difference in the number of surviving OT neurons in the SON or PVN (Fig. 6a and b). Moreover, quantitative analysis demonstrated that pharmacological inhibition of NF- $\mathrm{kB}$ with bay 11-7082 did not result in a significant difference in the proportional area of OT-immunoreactive processes in the SON compared to the $25 \mathrm{ng} / \mathrm{ml} \mathrm{rrCNTF}$ group (data not shown). When organotypic cultures were treated with bay 11-7082 in the absence of rrCNTF there was no difference in the number of OT neurons in the SON. In contrast, when organotypic

exogenous rrCNTF there was a significant increase of $112 \%$ in the proportional area of OT-immunoreactive processes compared to control $(p<0.0001)$. The LY303511 molecule served as a control molecule for the LY294002 inhibitor and the proportional area of OT-immunoreactive processes in the LY303511 in conjunction with $25 \mathrm{ng} / \mathrm{ml}$ rrCNTF group did not differ from the $25 \mathrm{ng} / \mathrm{ml} \mathrm{rrCNTF}$ group $(p=0.6496)$. However, when the PI3K inhibitors, LY294002 and wortmannin, were administered to the organotypic cultures in the presence of $25 \mathrm{ng} / \mathrm{ml} \mathrm{rrCNTF}$, there was a significant reduction in the proportional area of OT-immunoreactive processes in the SON compared to the $25 \mathrm{ng} / \mathrm{ml} \operatorname{rrCNTF}$ group $(p<$ $0.0001)$. Column bars and error bars represent the mean and SD of [n] groups. Scale bar $=100 \mu \mathrm{m} .{ }^{* *} p<0.01, * * * p<0.0001$

cultures were treated with sc-514 in the absence of $\operatorname{rrCNTF}$ a 181 and $166 \%$ increase in the number of OT neurons in the SON and PVN, respectively was observed indicating that activation of the NF- $\mathrm{KB}$ complex via a specific IKK $\beta$ component may promote neuronal loss.

\section{Discussion}

Our results indicate that MAPK, PI3-Akt and Jak-Stat pathways are activated in response to exogenous CNTF and these pathways mediate differentially neuronal 
survival and axonal regeneration by magnocellular neurons. While the Jak-STAT pathway is considered the canonical CNTF signal transduction pathway, CNTF has also been demonstrated to activate other intracellular signaling pathways including, MAPK, PI3K, and NFkB (Bonni et al. 1993; Cagnon and Braissant 2009; Dolcet et al. 2001; Gallagher et al. 2007; Kassen et al. 2009; Loy et al. 2011; Lutticken et al. 1994; Muller et al. 2009; Park et al. 2004; Peterson et al. 2000; Rhee et al. 2004; Sango et al. 2008; Symes et al. 1994; Trimarchi et al. 2009). For example, CNTF activates the MAPK pathway via SH2 domain-containing proteins, including SHP2 and Shc, which are bound to the LIFR and gp130 components of the CNTF receptor complex (Giordano et al. 1997; Stahl et al. 1995). Consistent with our findings others have demonstrated that the MAPK-ERK $1 \frac{1}{2}$ pathway promotes neuronal survival following injury (Chicoine and Bahr 2007; Jover-Mengual et al. 2007; Nagata 1999), primarily through the inhibition of the pro-apoptotic molecule $\mathrm{BAD}$ and production of the anti-apoptotic molecules, Bcl-2 and Bcl- $\mathrm{x}_{\mathrm{L}}$ (Nagata 1999). Our studies extend these observations by demonstrating that the MAPK-ERK $1 \frac{1}{2}$ pathway mediates CNTFinduced neuronal survival in the magnocellular neurosecretory system in vitro. Functional activation of the

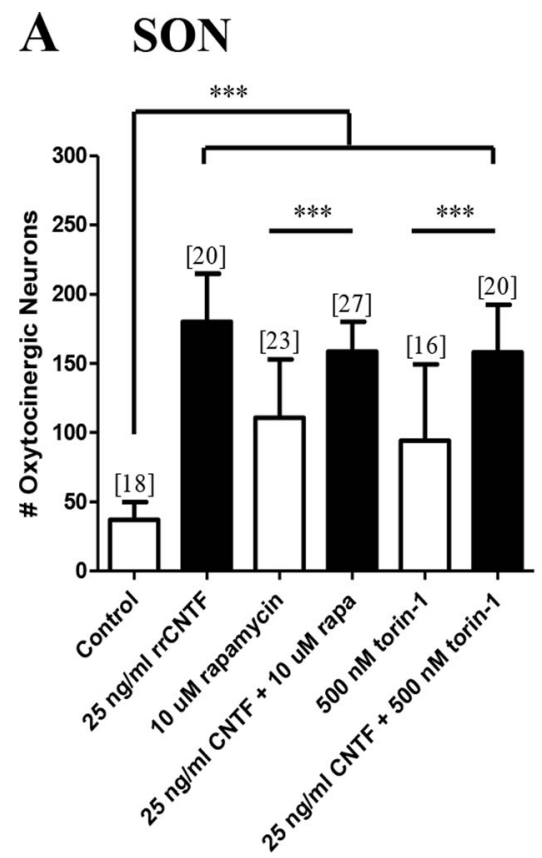

Fig. 5 mTOR is not necessary to mediate CNTF-induced survival of OT neurons. Immunohistochemical neuronal cell counts demonstrated that exogenous rrCNTF promoted the survival of OT neurons (SON: $p<$ 0.0001 ; PVN: $p<0.0001)$ compared to control, while inhibition of mTOR with rapamycin (SON: $p=0.1113$; PVN: $p=0.6633$ ) and torin-1 (SON: $p=0.1487$; PVN: $p=0.4250$ ) did not affect the number of surviving OT neurons in the SON (a) or PVN (b) compared to $25 \mathrm{ng} / \mathrm{ml} \mathrm{rCNTF}$.
MAPK-ERK $1 / 2$ pathway may occur via two potential mechanisms. First, unlike Jak molecules, which are tyrosine kinases, members of the MAPK pathway are serine/ threonine kinases. Thus, MAPK-dependent activation of STAT3 occurs at $\mathrm{Ser}^{727}$ as opposed to the more commonly Jak-dependently phosphorylated $\mathrm{Tyr}^{705}$ (Decker and Kovarik 2000). However, following pressure injection of exogenous rrCNTF in to the SON, we observed STAT3 activation specifically at the $\mathrm{Tyr}^{705}$ residue (Askvig et al. 2013) indicating that MAPK-ERK $1 \frac{1}{2}$ pathway is not upstream of STAT3 activation in the astrocytes. Alternatively, activation of the Jak-STAT pathway may lead to activation of the MAPK-ERK $1 / 2$ pathway (Frank et al. 1995; Ihle and Kerr 1995; Winston and Hunter 1996). This mechanism appears to be dependent on Jak-mediated Ras or Raf activation of ERK $1 \frac{1}{2}$. While Jak-mediated activation of STAT molecules has been reported to be Ras-independent, Jak-mediated activation of the MAPK-ERK $1 \frac{1}{2}$ pathway has been demonstrated to be Ras-dependent (Winston and Hunter 1995). Others have demonstrated that Raf physically associates with Jak2, and Raf is tyrosine phosphorylated when co-expressed with Jak2 (Xia et al. 1996) raising the possibility of CNTF activation of both pathways in parallel following exogenous CNTF.

\section{B PVN}

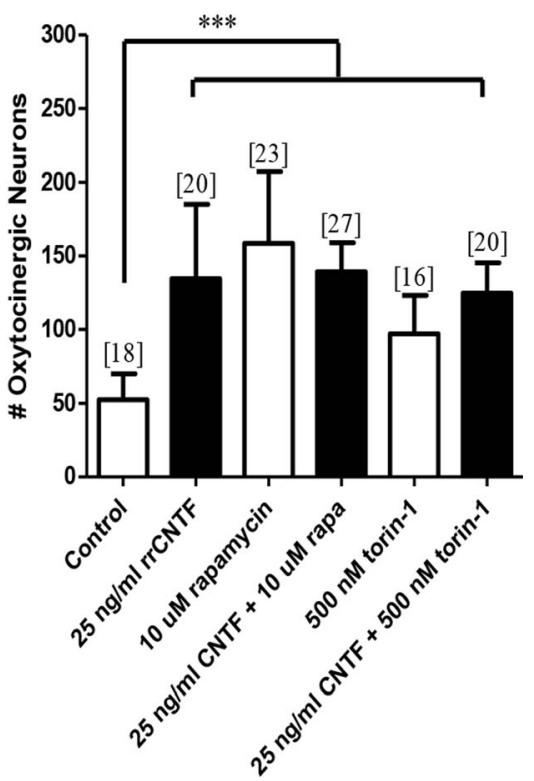

However, when cultures were treated with the inhibitors alone, there was a significant increase in the number of surviving OT neurons in the SON (A; rapamycin: $p<0.0001$; torin-1: $p<0.0001$ ) and PVN (B; rapamycin: $p<0.0001$; torin-1: $p<0.0001)$ compared to control. Column bars and error bars represent the mean and SD of [n] groups. $P V N$ paraventricular nucleus, $S O N$ supraoptic nucleus. ${ }^{* * *} p<0.0001$ 


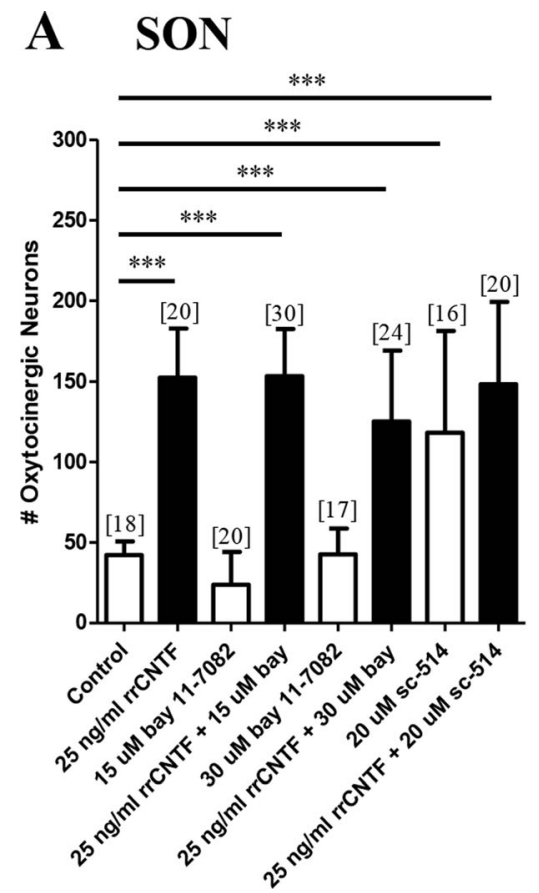

Fig. 6 NF- $k B$ is not necessary to mediate the CNTF-induced survival of OT neurons. Immunohistochemical neuronal cell counts demonstrated that exogenous rrCNTF promoted the survival of OT neurons (SON: $p$ $<0.0001$; PVN: $p<0.0001)$ compared to control, while inhibition of NF-кB with bay 11-7082 (15 $\mu \mathrm{M}$ : SON: $p=0.9228$; PVN: $p=0.9003$; $30 \mu \mathrm{M}$ : SON: $p=0.0828$; PVN: $p=0.0667$ ) and sc-514 (SON: $p=$ 0.7561 ; PVN: $p=0.3522$ ) did not affect the number of surviving OT

The role of the p38-, JNK-MAPK, and mTOR pathways in injury-induced neuronal death

The p38- and JNK-MAPK pathways are structurally similar, but functionally distinct, from the classic MAPKs (ERKs). These pathways are preferentially activated by environmental stresses such as UV radiation, heat and osmotic shock, and by pro-inflammatory cytokines, such as tumor necrosis factor (TNF) and interleukin-1 (IL-1) (Tibbles and Woodgett 1999). Others have demonstrated that CNTF, which is classified as a pro-inflammatory cytokine, is capable of activating the p38- (Loy et al. 2011) and the JNK-MAPK pathways (Cagnon and Braissant 2009). Pharmacological inhibition of the p38- and JNKMAPK pathways did not affect CNTF-mediated neuronal survival in hypothalamic organotypic cultures. However, unexpectedly, when the organotypic cultures were cultured only in the presence of the inhibitors, there was an increase in the survival of OT neurons. Similarly, when the organotypic cultures were cultured only in the presence of the mTOR inhibitors we observed a similar increase in the survival of OT neurons. These data suggest that the $\mathrm{p} 38$, JNK, and mTOR signaling components may mediate the post-axotomy responses that lead to neuronal death in the organotypic cultures.

\section{B PVN}

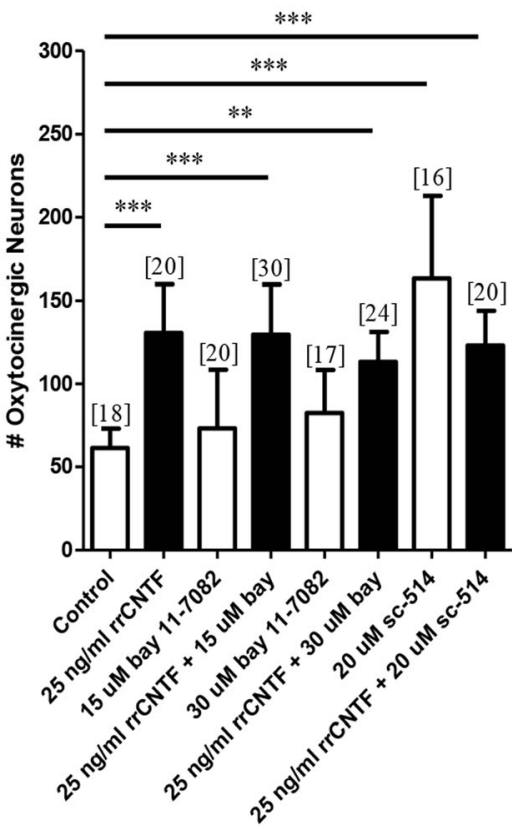

neurons in the SON (a) or PVN (b). However, when cultures were treated with sc-514 alone, there was a statistically significant increase in the number of surviving OT neurons in the SON $(\mathrm{A} ; p<0.0001)$ and PVN (B; $p<0.0001)$, compared to control. Column bars and error bars represent the mean and SD of [n] groups. $P V N$ paraventricular nucleus, $S O N$ supraoptic nucleus. $* * p<0.01, * * * p<0.0001$

Magnocellular vasopressinergic neurons in organotypic cultures of the PVN undergo neuronal death via apoptosis (Vutskits et al. 1998). Preparation of the organotypic cultures used in these studies resulted in a loss of greater than $90 \%$ of OT neurons (Askvig et al. 2013). Furthermore, the antiapoptotic agents, Bcl- $\mathrm{x}_{\mathrm{L}}$, and Z-VAD-fmk protect both OT and VP magnocellular neurons in the SON of organotypic cultures, (House et al. 2006). These data suggest that the loss of magnocellular neurons observed in our cultures is likely due to apoptosis. A well documented function of the p38MAPK and JNK-MAPK pathways is their role in the onset of apoptosis (Cuenda and Rousseau 2007; Dhanasekaran and Reddy 2008). Moreover, because of the overlap of the MAPK Ks, specifically MKK4 (Cargnello and Roux 2011), in the p38-MAPK and JNK-MAPK pathways, these pathways could be activated by the same stimulus. Thus, our data suggest that the enhanced survival of the OT neurons that we observed in the presence of the p38 and JNK inhibitors alone results from pharmacological inhibition of the apoptotic cascade.

We show that inhibition of mTOR also protects injured OT neurons in the absence of exogenous rrCNTF. While mTOR has been primarily linked to the PI3K-AKT pathway, numerous reports have demonstrated mTOR signaling in the MAPK pathways (Chen et al. 2008; Chen et al. 2011; Karassek et al. 2010; Kato et al. 2012; Miller et al. 2007; Xu et al. 2011). The 
primary functions of PI3K-AKT-mTOR signaling have been attributed to promoting process outgrowth, cell survival, proliferation and growth (Chen et al. 2008; Chen et al. 2011; Karassek et al. 2010; Kato et al. 2012; Miller et al. 2007; Xu et al. 2011; Zhou and Huang 2010). Paradoxically, mTORC1 signaling has also been shown to mediate apoptosis through inhibition of AKT and selective activation of the JNK-MAPK pathway (Kato et al. 2012). These reports suggest that JNK is capable of mediating an apoptotic response via mTOR, leading to our hypothesis that following the axotomy during the organotypic culture preparation the p38- and JNK-MAPK pathways were co-activated to induce an apoptotic cascade in the OT neurons through the downstream signaling of mTOR.

Within the magnocellular neurosecretory system, understanding the specific mechanisms that regulate injuryinduced neuronal apoptosis has not been well characterized. These novel data provide valuable insight into the mechanisms of axotomy-induced cell death that may lead to therapies that promote neuronal survival. Until future in vivo analyses can be performed, the full therapeutic potential remains unclear.

The role of the PI3K-AKT pathway in CNTF-induced process outgrowth

In addition to activating the Jak-STAT and MAPK pathways, reports have also demonstrated that CNTF activates the PI3KAKT pathway (Dolcet et al. 2001; Gold et al. 1994; Oh et al. 1998) through a Jak-dependent mechanism (Dolcet et al. 2001). Pharmacological inhibition of PI3K did not affect CNTF-induced neuronal survival, indicating that CNTF does not utilize PI3K signaling to promote OT neuronal survival in the magnocellular neurosecretory system. Interestingly, there was a remarkable decrease in process outgrowth in the SON following pharmacological inhibition of PI3K compared to the CNTF-treated cultures. Although the tissue thickness and complexity of the processes prevented stereological analysis of aspects of neurite outgrowth such as elongation, branching, and neurite caliber, we utilized quantitative optical densitometric stereological analysis to determine the proportional area of OT-immunoreactive processes which was corrected for the total number of neurons in the SON. This analysis resulted in a quantitative, yet conservative, analysis of processimmunoreactivity which confirmed our visual observation that there were less OT-immunoreactive processes present in the SON following PI3K inhibition. Thus, these data demonstrate that PI3K signaling is involved in regulating CNTFmediated process outgrowth, but not involved in mediating neuronal survival.

It is not surprising that diverse intracellular signaling pathways mediate distinct neuroprotective processes in response to CNTF. Numerous reports have demonstrated divergent pathway mediation of CNTF-induced neuronal survival and process outgrowth (Dolcet et al. 2001; Ozog et al. 2008; Park et al. 2004; Sango et al. 2008). Similarities between those reports and our own observations indicate that the PI3KAKT pathway plays a predominant role in mediating CNTFinduced process outgrowth in a variety of neuronal phenotypes. The PI3K-AKT pathway has been well-documented to promote neuronal survival, however, more recently the PI3K-AKT pathway has been revealed to be a key regulator in several aspects of process outgrowth, including elongation, branching, and neurite caliber (Read and Gorman 2009). There are many signaling factors downstream of PI3K that have been demonstrated to influence process outgrowth, including; mTORC1 (Asnaghi et al. 2004; Nave et al. 1999) and NF-kB (Kane et al. 1999; Romashkova and Makarov 1999). Not surprisingly, the transcriptional products of the PI3KAKT pathway that directly mediate process outgrowth are cytoskeletal elements, such as microtubules (Kobayashi et al. 1997).

mTOR functions as two distinct signaling complexes, mTORC1 and mTORC2. These two complexes consist of unique $\mathrm{mTOR}$-interacting proteins which determine their substrate specificity (Zhou and Huang 2010). Interestingly, the roles of the mTOR complexes in the PI3K-AKT pathway are quite distinct. It is believed that mTORC2 functions upstream of AKT to assist in regulating maximal activity of AKT (Hresko and Mueckler 2005; Sarbassov et al. 2005), while mTORC1 is a downstream regulator of AKT activity (Zhou and Huang 2010). It was demonstrated that PI3K-AKTmTOR signaling has been shown to promote growth and branching of hippocampal neurons (Jaworski et al. 2005). Our analysis demonstrated that pharmacological inhibition of mTORC1 and mTORC2 did not affect OT neuronal survival or the proportional area of OT-immunoreactive processes, suggesting that mTOR is not involved in CNTF-mediated PI3K-AKT dependent process outgrowth of OT neurons.

NF- $\mathrm{kB}$ has also been implicated in mediating process outgrowth downstream of the PI3K-AKT pathway (Armstrong et al. 2008; Gutierrez et al. 2005; O'Neill and Kaltschmidt 1997; Sole et al. 2004) therefore we sought to determine its potential role in mediating neuronal survival and process outgrowth in our system. Although our results show that inhibition of BAY-11-7082 and SC-514 had no affect on process outgrowth we did observe that inhibition of the IKK $\beta$ complex specifically and in the absence of CNTF resulted in a significant increase in cell survival in both the SON and $\mathrm{PVN}$. Increased NF-KB activation have been observed in neurons and astrocytes following CNS injury and evidence suggests a dual role for NF- $\mathrm{KB}$ in neuronal survival versus neuronal degeneration depending on the type of stimulus or cell phenotype activated (Mattson). Indeed, consistent with our observations, selective inactivation of NF- $\mathrm{KB}$ in astrocytes leads to increased survival of retinal ganglion cells following 
ischemia and promotes neuronal survival and axonal sprouting of central motor neurons following spinal cord injury (Brambilla2005.2009).

\section{Conclusions}

We and others have demonstrated previously that exogenous CNTF will induce magnocellular neuron sprouting in vitro (Askvig et al. 2013; Vutskits et al. 1998). However, the cellular signaling pathways mediating this event were not identified. In this study we have extended these observations to show that the PI3K pathway mediates CNTF-induced OT-process outgrowth in the SON. We also demonstrate here that the MAPK-ERK $1 \frac{1}{2}$ pathway promotes CNTF-induced neuronal survival. Furthermore, we show that the p38-, JNK-MAPK, mTOR and NF-kB pathways promote magnocellular neuronal survival in the absence of CNTF following axotomy. Taken together, these data indicate that distinct intracellular signaling pathways mediate diverse neuroprotective processes in response to CNTF. The results of these studies will provide the basis for future studies that will continue to elucidate the mechanisms underlying CNTF-mediated neuroprotection and axonal regeneration in the CNS. Our long-term goal is development of therapeutic strategies that will allow selective regulation of axonal sprouting and promote neuronal survival following injuries or disorders that result in neurodegeneration.

Acknowledgments We wish to thank Shirley House and Dr. Harold Gainer at the National Institutes for Health for their instruction on the hypothalamic organotypic explants and their gift of the OT-neurophysin antibodies. This publication was made possible by grant number P20RR017699 from the National Center for Research Resources (NCRR), a component of the National Institutes of Health (NIH) (JW) and the Doctoral Student Research Support Program (UND Graduate School) (JA).

Open Access This article is distributed under the terms of the Creative Commons Attribution License which permits any use, distribution, and reproduction in any medium, provided the original author(s) and the source are credited.

\section{References}

Alonzi T, Middleton G, Wyatt S, Buchman V, Betz UA, Muller W, Musiani P, Poli V, Davies AM (2001) Role of STAT3 and PI 3kinase/Akt in mediating the survival actions of cytokines on sensory neurons. Mol Cell Neurosci 18:270-282

Armstrong SJ, Wiberg M, Terenghi G, Kingham PJ (2008) Laminin activates NF-kappaB in Schwann cells to enhance neurite outgrowth. Neurosci Lett 439:42-46

Askvig JM, Leiphon LJ, Watt JA (2012) Neuronal activity and axonal sprouting differentially regulate CNTF and CNTF receptor complex in the rat supraoptic nucleus. Exp Neurol 233:243-252

Askvig JM, Lo DY, Sudbeck AW, Behm KE, Leiphon LJ, Watt JA (2013) Inhibition of the Jak-STAT pathway prevents CNTF-mediated survival of axotomized oxytocinergic magnocellular neurons in organotypic cultures of the rat supraoptic nucleus. Exp Neurol 240:75-87

Asnaghi L, Calastretti A, Bevilacqua A, D’Agnano I, Gatti G, Canti G, Delia D, Capaccioli S, Nicolin A (2004) Bcl-2 phosphorylation and apoptosis activated by damaged microtubules require mTOR and are regulated by Akt. Oncogene 23:5781-5791

Bain J, Plater L, Elliott M, Shpiro N, Hastie CJ, McLauchlan H, Klevernic I, Arthur JS, Alessi DR, Cohen P (2007) The selectivity of protein kinase inhibitors: a further update. Biochem J 408:297315

Ballif BA, Blenis J (2001) Molecular mechanisms mediating mammalian mitogen-activated protein kinase (MAPK) kinase (MEK)-MAPK cell survival signals. Cell Growth Differ: Mol Biol J Am Assoc Cancer Res 12:397-408

Ben-Barak Y, Russell JT, Whitnall MH, Ozato K, Gainer H (1985) Neurophysin in the hypothalamo-neurohypophysial system. I. Production and characterization of monoclonal antibodies. J Neurosci: Off J Soc Neurosci 5:81-97

Bennett BL, Sasaki DT, Murray BW, O’Leary EC, Sakata ST, Xu W, Leisten JC, Motiwala A, Pierce S, Satoh Y, Bhagwat SS, Manning AM, Anderson DW (2001) SP600125, an anthrapyrazolone inhibitor of Jun N-terminal kinase. Proc Natl Acad Sci U S A 98:1368113686

Bilsland J, Rigby M, Young L, Harper S (1999) A rapid method for semiquantitative analysis of neurite outgrowth from chick DRG explants using image analysis. J Neurosci Methods 92:75-85

Bonni A, Frank DA, Schindler C, Greenberg ME (1993) Characterization of a pathway for ciliary neurotrophic factor signaling to the nucleus. Science 262:1575-1579

Cagnon L, Braissant O (2009) CNTF protects oligodendrocytes from ammonia toxicity: intracellular signaling pathways involved. Neurobiol Dis 33:133-142

Cargnello M, Roux PP (2011) Activation and function of the MAPKs and their substrates, the MAPK-activated protein kinases. Microbiol Mol Biol Rev: MMBR 75:50-83

Chen L, Liu L, Luo Y, Huang S (2008) MAPK and mTOR pathways are involved in cadmium-induced neuronal apoptosis. J Neurochem 105:251-261

Chen S, Xu Y, Xu B, Guo M, Zhang Z, Liu L, Ma H, Chen Z, Luo Y, Huang S, Chen L (2011) CaMKII is involved in cadmium activation of MAPK and mTOR pathways leading to neuronal cell death. J Neurochem 119:1108-1118

Chicoine LM, Bahr BA (2007) Excitotoxic protection by polyanionic polysaccharide: evidence of a cell survival pathway involving AMPA receptor-MAPK Interactions. J Neurosci Res 85:294-302

Cuenda A, Rousseau S (2007) p38 MAP-kinases pathway regulation, function and role in human diseases. Biochim Biophys Acta 1773: $1358-1375$

Davis S, Aldrich TH, Stahl N, Pan L, Taga T, Kishimoto T, Ip NY, Yancopoulos GD (1993) LIFR beta and gp130 as heterodimerizing signal transducers of the tripartite CNTF receptor. Science 260: 1805-1808

Decker T, Kovarik P (2000) Serine phosphorylation of STATs. Oncogene 19:2628-2637

Dhanasekaran DN, Reddy EP (2008) JNK signaling in apoptosis. Oncogene 27:6245-6251

Dolcet X, Soler RM, Gould TW, Egea J, Oppenheim RW, Comella JX (2001) Cytokines promote motoneuron survival through the Janus kinase-dependent activation of the phosphatidylinositol 3-kinase pathway. Mol Cell Neurosci 18:619-631

English JM, Cobb MH (2002) Pharmacological inhibitors of MAPK pathways. Trends Pharmacol Sci 23:40-45

Frank SJ, Yi W, Zhao Y, Goldsmith JF, Gilliland G, Jiang J, Sakai I, Kraft AS (1995) Regions of the JAK2 tyrosine kinase required for 
coupling to the growth hormone receptor. J Biol Chem 270:1477614785

Gallagher D, Gutierrez H, Gavalda N, O'Keeffe G, Hay R, Davies AM (2007) Nuclear factor-kappaB activation via tyrosine phosphorylation of inhibitor kappaB-alpha is crucial for ciliary neurotrophic factor-promoted neurite growth from developing neurons. J Neurosci 27:9664-9669

Giordano V, De Falco G, Chiari R, Quinto I, Pelicci PG, Bartholomew L, Delmastro P, Gadina M, Scala G (1997) Shc mediates IL-6 signaling by interacting with gp130 and Jak2 kinase. J Immunol 158:40974103

Gold MR, Duronio V, Saxena SP, Schrader JW, Aebersold R (1994) Multiple cytokines activate phosphatidylinositol 3-kinase in hemopoietic cells. Association of the enzyme with various tyrosinephosphorylated proteins. J Biol Chem 269:5403-5412

Greenwood SM, Bushell TJ (2010) Astrocytic activation and an inhibition of MAP kinases are required for proteinase-activated receptor2-mediated protection from neurotoxicity. J Neurochem 113:14711480

Gurney ME, Yamamoto H, Kwon Y (1992) Induction of motor neuron sprouting in vivo by ciliary neurotrophic factor and basic fibroblast growth factor. J Neurosci: Off J Soc Neurosci 12:3241-3247

Guthrie KM, Woods AG, Nguyen T, Gall CM (1997) Astroglial ciliary neurotrophic factor mRNA expression is increased in fields of axonal sprouting in deafferented hippocampus. J Comp Neurol 386: 137-148

Gutierrez H, Hale VA, Dolcet X, Davies A (2005) NF-kappaB signalling regulates the growth of neural processes in the developing PNS and CNS. Development 132:1713-1726

House SB, Thomas A, Kusano K, Gainer H (1998) Stationary organotypic cultures of oxytocin and vasopressin magnocellular neurones from rat and mouse hypothalamus. J Neuroendocrinol 10:849-861

House SB, Rusnak M, Liu XH, Youle RJ, Gainer H (2006) Bcl-xL and caspase inhibition increase the survival of rat oxytocin and vasopressin magnocellular neurons in organotypic culture. Exp Neurol 200: 267-271

House SB, Li C, Yue C, Gainer H (2009) Effects of ciliary neurotrophic factor and leukemia inhibiting factor on oxytocin and vasopressin magnocellular neuron survival in rat and mouse hypothalamic organotypic cultures. J Neurosci Methods 178:128-133

Hresko RC, Mueckler M (2005) mTOR.RICTOR is the Ser473 kinase for Akt/protein kinase B in 3T3-L1 adipocytes. J Biol Chem 280: 40406-40416

Ihle JN, Kerr IM (1995) Jaks and Stats in signaling by the cytokine receptor superfamily. Trends Genet: TIG 11:69-74

Ip NY, Nye SH, Boulton TG, Davis S, Taga T, Li Y, Birren SJ, Yasukawa K, Kishimoto T, Anderson DJ et al (1992) CNTF and LIF act on neuronal cells via shared signaling pathways that involve the IL-6 signal transducing receptor component gp130. Cell 69:1121-1132

Ip NY, McClain J, Barrezueta NX, Aldrich TH, Pan L, Li Y, Wiegand SJ, Friedman B, Davis S, Yancopoulos GD (1993) The alpha component of the CNTF receptor is required for signaling and defines potential CNTF targets in the adult and during development. Neuron 10:89-102

Itoh K, Konishi A, Nomura S, Mizuno N, Nakamura Y, Sugimoto T (1979) Application of coupled oxidation reaction to electron microscopic demonstration of horseradish peroxidase: cobalt-glucose oxidase method. Brain Res 175:341-346

Jaworski J, Spangler S, Seeburg DP, Hoogenraad CC, Sheng M (2005) Control of dendritic arborization by the phosphoinositide-3'-kinaseAkt-mammalian target of rapamycin pathway. J Neurosci: Off J Soc Neurosci 25:11300-11312

Jover-Mengual T, Zukin RS, Etgen AM (2007) MAPK signaling is critical to estradiol protection of CA1 neurons in global ischemia. Endocrinology 148:1131-1143
Kane LP, Shapiro VS, Stokoe D, Weiss A (1999) Induction of NFkappaB by the Akt/PKB kinase. Curr Biol: CB 9:601-604

Karassek S, Berghaus C, Schwarten M, Goemans CG, Ohse N, Kock G, Jockers K, Neumann S, Gottfried S, Herrmann C, Heumann R, Stoll R (2010) Ras homolog enriched in brain (Rheb) enhances apoptotic signaling. J Biol Chem 285:33979-33991

Kassen SC, Thummel R, Campochiaro LA, Harding MJ, Bennett NA, Hyde DR (2009) CNTF induces photoreceptor neuroprotection and Muller glial cell proliferation through two different signaling pathways in the adult zebrafish retina. Exp Eye Res 88:1051-1064

Kato H, Nakajima S, Saito Y, Takahashi S, Katoh R, Kitamura M (2012) mTORC1 serves ER stress-triggered apoptosis via selective activation of the IRE1-JNK pathway. Cell Death Differ 19:310-320

Kobayashi M, Nagata S, Kita Y, Nakatsu N, Ihara S, Kaibuchi K, Kuroda S, Ui M, Iba H, Konishi H, Kikkawa U, Saitoh I, Fukui Y (1997) Expression of a constitutively active phosphatidylinositol 3-kinase induces process formation in rat $\mathrm{PC} 12$ cells. Use of Cre/loxP recombination system. J Biol Chem 272:16089-16092

Kwon YW, Gurney ME (1994) Systemic injections of ciliary neurotrophic factor induce sprouting by adult motor neurons. Neuroreport 5: 789-792

Lee JC, Laydon JT, McDonnell PC, Gallagher TF, Kumar S, Green D, McNulty D, Blumenthal MJ, Heys JR, Landvatter SW et al (1994) A protein kinase involved in the regulation of inflammatory cytokine biosynthesis. Nature 372:739-746

Loy B, Apostolova G, Dorn R, McGuire VA, Arthur JS, Dechant G (2011) p38alpha and p38beta mitogen-activated protein kinases determine cholinergic transdifferentiation of sympathetic neurons. J Neurosci: Off J Soc Neurosci 31:12059-12067

Luo JM, Cen LP, Zhang XM, Chiang SW, Huang Y, Lin D, Fan YM, van Rooijen N, Lam DS, Pang CP, Cui Q (2007) PI3K/akt, JAK/STAT and MEK/ERK pathway inhibition protects retinal ganglion cells via different mechanisms after optic nerve injury. Eur J Neurosci 26: $828-842$

Lutticken C, Wegenka UM, Yuan J, Buschmann J, Schindler C, Ziemiecki A, Harpur AG, Wilks AF, Yasukawa K, Taga T et al (1994) Association of transcription factor APRF and protein kinase Jak1 with the interleukin-6 signal transducer gp130. Science 263: 89-92

Marwarha G, Dasari B, Prabhakara JP, Schommer J, Ghribi O (2010) beta-Amyloid regulates leptin expression and tau phosphorylation through the mTORC1 signaling pathway. J Neurochem 115:373384

Miller AL, Garza AS, Johnson BH, Thompson EB (2007) Pathway interactions between MAPKs, mTOR, PKA, and the glucocorticoid receptor in lymphoid cells. Cancer Cell Int 7:3

Muller A, Hauk TG, Leibinger M, Marienfeld R, Fischer D (2009) Exogenous CNTF stimulates axon regeneration of retinal ganglion cells partially via endogenous CNTF. Mol Cell Neurosci 41:233246

Nagata S (1999) Fas ligand-induced apoptosis. Annu Rev Genet 33:2955

Nave BT, Ouwens M, Withers DJ, Alessi DR, Shepherd PR (1999) Mammalian target of rapamycin is a direct target for protein kinase B: identification of a convergence point for opposing effects of insulin and amino-acid deficiency on protein translation. Biochem J 344(Pt 2):427-431

Oh H, Fujio Y, Kunisada K, Hirota H, Matsui H, Kishimoto T, YamauchiTakihara K (1998) Activation of phosphatidylinositol 3-kinase through glycoprotein 130 induces protein kinase B and p70 S6 kinase phosphorylation in cardiac myocytes. J Biol Chem 273:97039710

Ohnishi M, Katsuki H, Izumi Y, Kume T, Takada-Takatori Y, Akaike A (2010) Mitogen-activated protein kinases support survival of activated microglia that mediate thrombin-induced striatal injury in organotypic slice culture. J Neurosci Res 88:2155-2164 
O’Neill LA, Kaltschmidt C (1997) NF-kappa B: a crucial transcription factor for glial and neuronal cell function. Trends Neurosci 20:252258

Oyesiku NM, Wigston DJ (1996) Ciliary neurotrophic factor stimulates neurite outgrowth from spinal cord neurons. J Comp Neurol 364: 68-77

Ozog MA, Modha G, Church J, Reilly R, Naus CC (2008) Coadministration of ciliary neurotrophic factor with its soluble receptor protects against neuronal death and enhances neurite outgrowth. J Biol Chem 283:6546-6560

Park K, Luo JM, Hisheh S, Harvey AR, Cui Q (2004) Cellular mechanisms associated with spontaneous and ciliary neurotrophic factorcAMP-induced survival and axonal regeneration of adult retinal ganglion cells. J Neurosci: Off J Soc Neurosci 24:10806-10815

Peterson WM, Wang Q, Tzekova R, Wiegand SJ (2000) Ciliary neurotrophic factor and stress stimuli activate the Jak-STAT pathway in retinal neurons and glia. J Neurosci: Off J Soc Neurosci 20:40814090

Phulwani NK, Esen N, Syed MM, Kielian T (2008) TLR2 expression in astrocytes is induced by TNF-alpha- and NF-kappa B-dependent pathways. J Immunol 181:3841-3849

Read DE, Gorman AM (2009) Involvement of Akt in neurite outgrowth. Cell Mol Life Sci: CMLS 66:2975-2984

Rhee KD, Goureau O, Chen S, Yang XJ (2004) Cytokine-induced activation of signal transducer and activator of transcription in photoreceptor precursors regulates rod differentiation in the developing mouse retina. J Neurosci: Off J Soc Neurosci 24:9779-9788

Romashkova JA, Makarov SS (1999) NF-kappaB is a target of AKT in anti-apoptotic PDGF signalling. Nature 401:86-90

Rusnak M, Gainer H (2005) Differential effects of forskolin on tyrosine hydroxylase gene transcription in identified brainstem catecholaminergic neuronal subtypes in organotypic culture. Eur $\mathrm{J}$ Neurosci 21:889-898

Rusnak M, House SB, Arima H, Gainer H (2002) Ciliary neurotrophic factor increases the survival of magnocellular vasopressin and oxytocin neurons in rat supraoptic nucleus in organotypic cultures. Microsc Res Tech 56:101-112

Rusnak M, House SB, Gainer H (2003) Long-term effects of ciliary neurotrophic factor on the survival of vasopressin magnocellular neurones in the rat supraoptic nucleus in vitro. J Neuroendocrinol 15:933-939

Sango K, Yanagisawa H, Komuta Y, Si Y, Kawano H (2008) Neuroprotective properties of ciliary neurotrophic factor for cultured adult rat dorsal root ganglion neurons. Histochem Cell Biol 130: 669-679

Sarbassov DD, Guertin DA, Ali SM, Sabatini DM (2005) Phosphorylation and regulation of Akt/PKB by the rictor-mTOR complex. Science 307:1098-1101

Schuster B, Kovaleva M, Sun Y, Regenhard P, Matthews V, Grotzinger J, Rose-John S, Kallen KJ (2003) Signaling of human ciliary neurotrophic factor (CNTF) revisited. The interleukin-6 receptor can serve as an alpha-receptor for CTNF. J Biol Chem 278:9528-9535

Shahar T, House SB, Gainer H (2004) Neural activity protects hypothalamic magnocellular neurons against axotomy-induced programmed cell death. J Neurosci: Off J Soc Neurosci 24:6553-6562

Siegel SG, Patton B, English AW (2000) Ciliary neurotrophic factor is required for motoneuron sprouting. Exp Neurol 166:205-212

Simon CM, Jablonka S, Ruiz R, Tabares L, Sendtner M (2010) Ciliary neurotrophic factor-induced sprouting preserves motor function in a mouse model of mild spinal muscular atrophy. Hum Mol Genet 19: 973-986

Sole C, Dolcet X, Segura MF, Gutierrez H, Diaz-Meco MT, Gozzelino R, Sanchis D, Bayascas JR, Gallego C, Moscat J, Davies AM, Comella JX (2004) The death receptor antagonist FAIM promotes neurite outgrowth by a mechanism that depends on ERK and NF-kapp B signaling. J Cell Biol 167:479-492
Stahl N, Farruggella TJ, Boulton TG, Zhong Z, Darnell JE Jr, Yancopoulos GD (1995) Choice of STATs and other substrates specified by modular tyrosine-based motifs in cytokine receptors. Science 267:1349-1353

Stoppini L, Buchs PA, Muller D (1991) A simple method for organotypic cultures of nervous tissue. J Neurosci Methods 37:173-182

Su C, Underwood W, Rybalchenko N, Singh M (2011) ERK1/2 and ERK5 have distinct roles in the regulation of brain-derived neurotrophic factor expression. J Neurosci Res 89:1542-1550

Symes A, Lewis S, Corpus L, Rajan P, Hyman SE, Fink JS (1994) STAT proteins participate in the regulation of the vasoactive intestinal peptide gene by the ciliary neurotrophic factor family of cytokines. Mol Endocrinol 8:1750-1763

Thoreen CC, Sabatini DM (2009) Rapamycin inhibits mTORC1, but not completely. Autophagy 5:725-726

Thoreen CC, Kang SA, Chang JW, Liu Q, Zhang J, Gao Y, Reichling LJ, Sim T, Sabatini DM, Gray NS (2009) An ATP-competitive mammalian target of rapamycin inhibitor reveals rapamycin-resistant functions of mTORC1. J Biol Chem 284:8023-8032

Thoreen CC, Chantranupong L, Keys HR, Wang T, Gray NS, Sabatini DM (2012) A unifying model for mTORC1-mediated regulation of mRNA translation. Nature 485:109-113

Tibbles LA, Woodgett JR (1999) The stress-activated protein kinase pathways. Cell Mol Life Sci: CMLS 55:1230-1254

Trimarchi T, Pachuau J, Shepherd A, Dey D, Martin-Caraballo M (2009) CNTF-evoked activation of JAK and ERK mediates the functional expression of T-type $\mathrm{Ca} 2+$ channels in chicken nodose neurons. $\mathrm{J}$ Neurochem 108:246-259

Ulenkate HJ, Kaal EC, Gispen WH, Jennekens FG (1994) Ciliary neurotrophic factor improves muscle fibre reinnervation after facial nerve crush in young rats. Acta Neuropathol 88:558-564

Vutskits L, Bartanusz V, Schulz MF, Kiss JZ (1998) Magnocellular vasopressinergic neurons in explant cultures are rescued from cell death by ciliary neurotrophic factor and leukemia inhibiting factor. Neuroscience 87:571-582

Vutskits L, Gascon E, Kiss JZ (2003) Removal of PSA from NCAM affects the survival of magnocellular vasopressin- and oxytocinproducing neurons in organotypic cultures of the paraventricular nucleus. Eur J Neurosci 17:2119-2126

Wang JM, Liu L, Brinton RD (2008) Estradiol-17beta-induced human neural progenitor cell proliferation is mediated by an estrogen receptor beta-phosphorylated extracellularly regulated kinase pathway. Endocrinology 149:208-218

Watt JA, Bone S, Pressler M, Cranston HJ, Paden CM (2006) Ciliary neurotrophic factor is expressed in the magnocellular neurosecretory system of the rat in vivo: evidence for injury- and activity-induced upregulation. Exp Neurol 197:206-214

Watt JA, Lo D, Cranston HJ, Paden CM (2009) CNTF receptor alpha is expressed by magnocellular neurons and expression is upregulated in the rat supraoptic nucleus during axonal sprouting. Exp Neurol 215:135-141

Winston LA, Hunter T (1995) JAK2, Ras, and Raf are required for activation of extracellular signal-regulated kinase/mitogen-activated protein kinase by growth hormone. J Biol Chem 270:30837-30840

Winston LA, Hunter T (1996) Intracellular signalling: putting JAKs on the kinase MAP. Curr Biol: CB 6:668-671

Wright MC, Cho WJ, Son YJ (2007) Distinct patterns of motor nerve terminal sprouting induced by ciliary neurotrophic factor vs. botulinum toxin. J Comp Neurol 504:1-16

Xia K, Mukhopadhyay NK, Inhorn RC, Barber DL, Rose PE, Lee RS, Narsimhan RP, D'Andrea AD, Griffin JD, Roberts TM (1996) The cytokine-activated tyrosine kinase JAK2 activates Raf-1 in a p21ras-dependent manner. Proc Natl Acad Sci U S A 93:11681-11686

Xu JJ, Chen EY, Lu CL, He C (2009) Recombinant ciliary neurotrophic factor promotes nerve regeneration and induces gene 
expression in silicon tube-bridged transected sciatic nerves in adult rats. J Clin Neurosci: Off J Neurosurg Soc Australas 16: $812-817$

Xu B, Chen S, Luo Y, Chen Z, Liu L, Zhou H, Chen W, Shen T, Han X, Chen L, Huang S (2011) Calcium signaling is involved in cadmiuminduced neuronal apoptosis via induction of reactive oxygen species and activation of MAPK/mTOR network. PLoS One 6:e19052
Zamin LL, Dillenburg-Pilla P, Argenta-Comiran R, Horn AP, Simao F, Nassif M, Gerhardt D, Frozza RL, Salbego C (2006) Protective effect of resveratrol against oxygen-glucose deprivation in organotypic hippocampal slice cultures: Involvement of PI3-K pathway. Neurobiol Dis 24:170-182

Zhou H, Huang S (2010) The complexes of mammalian target of rapamycin. Curr Protein Pept Sci 11:409-424 